\title{
Decisive Factors of False Detection on Spectrum Sensing in Cognitive Radio
}

\author{
Lailun Nahar ${ }^{1}$, Sameha Zaman Ruthi ${ }^{2}$, Fakir Mashuque Alamgir ${ }^{3}$ and Anwarul Azim ${ }^{4}$ \\ ${ }^{1,2,3,4}$ East West University, Department of Electrical \& Electronic Engineering, Dhaka, Bangladesh

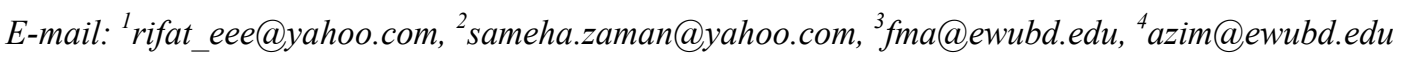

\begin{abstract}
The increasing demand of wireless communication introduces efficient spectrum utilization challenges. Cognitive radio has emerged as the key technology to address this challenge which enables opportunistic access to the spectrum. Cooperative spectrum sensing improves the probability of detection by collaborating to detect primary user's signal in cognitive radio (CR) system as well. The goal of this thesis is to cooperate the cognitive users to increase the detection probability for a given probability of false alarm and find out the better condition of sensing spectrum among three rules: AND, Majority, OR. It is observed that under the AWGN the detection probability for a given value of false alarm has been significantly achieved by optimal value of detection probability in the AND rule as the number of the user increases. Finally we have observed that due to fading condition, AND rule gives better performance in detection probability among three rules.
\end{abstract}

Keywords: Cooperative Sensing, Probability of False Alarm, Probability of Miss Detection, AWGN Channel, Fading Condition.

\section{INTRODUCTION}

Cognitive Radio (CR) is an adaptive, intelligent radio and network technology that can automatically detect available channels in a wireless spectrum and change transmission parameters enabling more communications to run concurrently and also can improve radio operating behavior. Cognitive radio networks (from now on called secondary networks) will also need to coexist with legacy ones which have the right to their spectrum slice and thus cannot accept interference.

2

\section{FEATURES OF COGNITIVE RADIO}

The followings are the features of cognitive radio:

- Lailun Nahar is with the Department of Electrical \& Electronic Engineering, East West University, Dhaka, Bangladesh.

- Sameha Zaman Ruthi is with LM Ericsson Ltd., Dhaka, Bangladesh.

- Fakir Mashuque Alamgir is with the Department of Electrical \& Electronic Engineering, East West University, Dhaka, Bangladesh.

- Anwarul Azim is with the Department of Electrical \& Electronic Engineering, East West University, Dhaka,

\subsection{Frequency Agility}

Cognitive radio has this ability to change its operating frequency to optimize the usage under certain conditions.

\subsection{Dynamic Frequency Selection (DFS)}

Cognitive radio has this ability to sense signals from other nearby transmitters in an effort to choose an optimum operating environment.

\subsection{Location Awareness}

CR provides the ability for a device to determine its location and the location of other transmitters. At first, it determines whether it is permissible to transmit at all, then to select the appropriate operating parameters such as the power and frequency allowed at its location.

\section{$2.4 \quad$ Negotiated Use}

A cognitive radio can incorporate a mechanism that enables the sharing of spectrum under the 
terms of a prearranged agreement between a licensee and a third party.

\subsection{Adaptive Modulation}

CR features with the ability to modify transmission characteristics and waveforms to exploit opportunities to use spectrum.

\subsection{Transmit Power Control (TPC)}

CR allows full power limits when it needs to transmit. But it can construct the transmitter power to a lower level to allow greater sharing of spectrum when higher power operation is not necessary [1].

\section{METHODOLOGY ADOPTED AND JUSTIFICATION}

We mainly focused on the justification of this paper based on cooperative sensing according to three rules which are AND, Majority, OR rules. Here, we have analyzed the Cooperative Spectrum Sensing under the AWGN channel. Cooperative Spectrum Sensing (CSS) can improve the spectrum sensing performance by introducing spatial diversity in cognitive radio networks (CRNs). In cooperative detection when each sensor takes the own decision and transmits only its binary number to the fusion center, the fusion center combines the hard decision to one common decision. Overall, we can say that all users transmit soft decision to a fusion center which combines the soft values to one common decision. If we think there are $\mathrm{M}$ sensors and wish to detect whether there is a signal present or not then we have to discriminate between the following two hypothesis tests.

H0: $y m=W m, \quad m=0, \ldots, M-1$

H1: $\quad y m=X m+W m, \quad m=0, \ldots, M-1$

Here $(\mathrm{ym})$ is the signal vector of length $m$ which consists of a signal pulse noise. In equation 2. (Xm) is the signal vector and $(\mathrm{Wm})$ is the noise vector.

If the received signals at all sensors are independent.

Let, $Z=(y T 0, y T 1 \ldots y T M-1) T$, then the loglikelihood ratio will be,

$$
\begin{aligned}
& \text { Acoop } \triangleq \log \left(\frac{P(z \mid H 1)}{P(z \mid H o)}\right) \\
& \text { Acoop }=\sum_{m=0}^{M-1} \log \left(\frac{P(y m \mid H 1)}{P(y m \mid H o)}\right) \\
& \text { Acoop }=\sum_{m=0}^{M-1} \mathrm{~A}(\mathbf{m})
\end{aligned}
$$

Here, $\mathrm{A}(\mathrm{m}) \triangleq \log ((\mathrm{P}(\mathrm{ym} \mid \mathrm{H} 1)) /(\mathrm{P}(\mathrm{ym} \mid \mathrm{Ho})))$ is the log- likelihood ratio for the mth sensor. It means that if all the sensors of receive signal is independent, then the soft combining of fusion rule is equal to sum of log-likelihood ratios so that we can say log- likelihood ratio depends on the distribution of the signal to be detected [2].

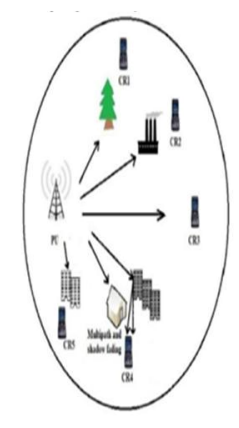

(a)

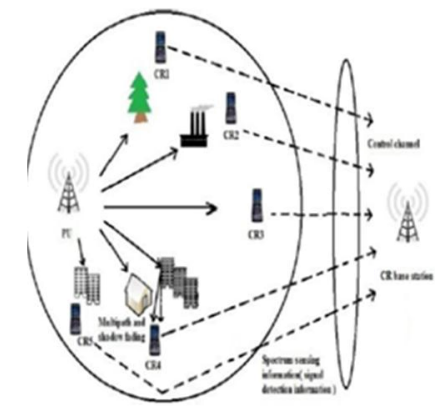

(b)
Fig. 1. (a) Non cooperative system and (b) Cooperative system for Cognitive Radio [2]

As shown in Figure 1 (a), CR users detect primary signal and decide whether the signal is present or not by themselves. However, this technique cannot detect primary signal properly due to fading and shadowing. Fig. 1(b), shows the system model of cooperative signal detection where only one cognitive radio user could be able to detect the primary signal.

Here the users are populated in the range of primary transmitter. So it is expected that it can improve the signal detection probability [3]. From the above Figures, we can understand that the cooperative sensing is an effective and attractive approach to combat multiple fading, receiver's uncertainty and hidden primary problem.

\subsection{Justification Behind Selecting Rule}

There are different rules for combining cooperative sensing such as AND, OR and VOITING / Majority rules. The OR rule decides the signal present if any of the sensor reports signal detection. So for the OR rule, the cooperative test decides on $\mathrm{H} 1$, if

$$
\sum_{m=0}^{M-1} A(m)=1
$$

Assume that the individual statistics A (m) are quantized to one bit such that $\mathrm{A}(\mathrm{m})=0,1$ is the hard decision from the mth sensor. In this case when a signal is detected then it becomes 1 otherwise it becomes 0 . 
The Majority Rule decides that a signal is present if at least $\mathrm{V}$ of the $\mathrm{M}$ sensor or user has detected a signal since 1.

$$
\sum_{m=0}^{M-1} A(m) \geq_{V}
$$

Here $\mathrm{V}$ is considered as $\mathrm{M} / 2$.

In AND-rule, If all sensors have detected a signal, then an AND rule decides that a signal is detected. So, here the cooperative test using the AND rule decides on $\mathrm{H} 1$, if

$$
\sum_{\mathrm{m}=0}^{\mathrm{M}-1} \mathrm{~A}(\mathrm{~m})=\mathrm{M}
$$

In our paper for the spectrum sensing of cooperative, we followed the AND rule because we wanted to see the effect on the probability of detection of the signal in the case of busty situation. In spectrum sensing there are two types of errors which are false alarming and miss-detection. We know that the probability of detection $(\mathrm{Pd})$ and probability of false alarm (Pfa) depend on the threshold value, noise variance and signal variance of the primary user when the SNR value is large. We can calculate the probability of detection $(\mathrm{Pd})$ and probability of false alarm (Pfa) by the following formula:

$$
P f a=Q\left(\frac{\sqrt{ }(\beta E D-\sigma 2 \mathrm{n})}{\sigma 2 \mathrm{n}}\right)
$$

Here,

$$
\begin{aligned}
& \sigma_{S}^{2}=\text { the primary user signal variance. } \\
& \sigma_{n}^{2}=\text { the noise variance } \\
& \beta_{E D}=\text { threshold value. }
\end{aligned}
$$

After the calculation of Pd and Pfa, we can calculate the probability of false alarm and probability of detection of the cognitive radio for the $\mathrm{M}$ user under AND rule.

$$
\begin{aligned}
\mathrm{Cd} & =\prod_{1}^{\mathrm{q}} \mathrm{Pd}, \mathrm{m} \\
\mathrm{Cfa} & =\prod_{1}^{q} \mathrm{Pfa}, \mathrm{m}
\end{aligned}
$$

For OR rule we can calculate the probability of false alarm and probability of detection by following formulas:

$$
\begin{array}{r}
\mathrm{Cd}=1-\prod_{1}^{\mathrm{q}} \mathrm{Pd}, \mathrm{m} \\
\mathrm{Cfa}=1-\prod_{1}^{\mathrm{q}} \mathrm{Pfa}, \mathrm{m}
\end{array}
$$

Here, $\mathrm{q}$ is the number of Cognitive radio. $\mathrm{Pd}, \mathrm{m}$ and $\mathrm{Pfa}, \mathrm{m}$ is the probability of detection and probability of false alarm or the $\mathrm{M}$ user respectively.

\section{RESULT ANALYSIS}

First of all, we simulated the miss detection for the fixed bandwidth factor. In Figure (2) we show the given SNR value is $15 \mathrm{~dB}$ and the time bandwidth factor is 1200 for the curve majority or voting rule.

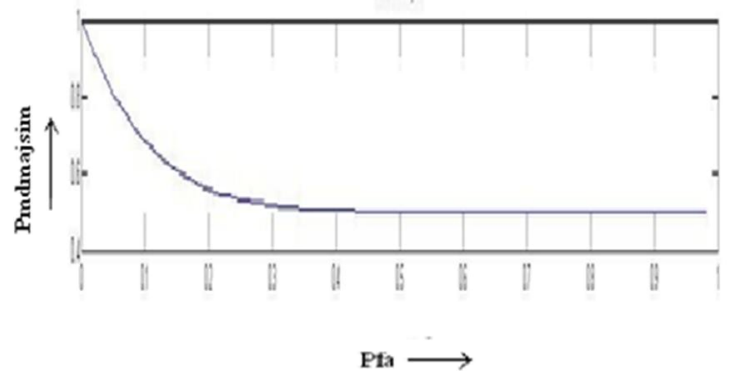

Fig. 2. Pfa vs Pmdmaj for bandwidth 1200. From this Figure 2, we see that the detection probability decreases with the increasing false alarm but it does not become zero

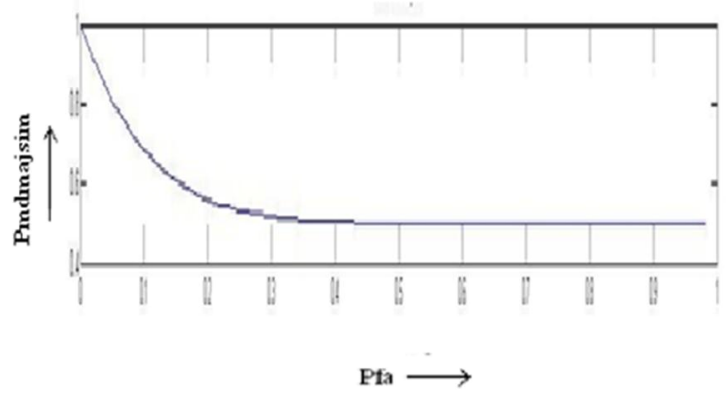

Fig. 3. Pfa vs Pmdmaj for bandwidth 2000

In Figure. (3) for the changing time bandwidth factor 1200 to 2000 in majority rule we can see that factors there is no effect on the probability of detection with the change of probability of false alarm. 
L. Nahar et al. / International Journal of Computer Networks and Communications Security, 1 (6), November 2013

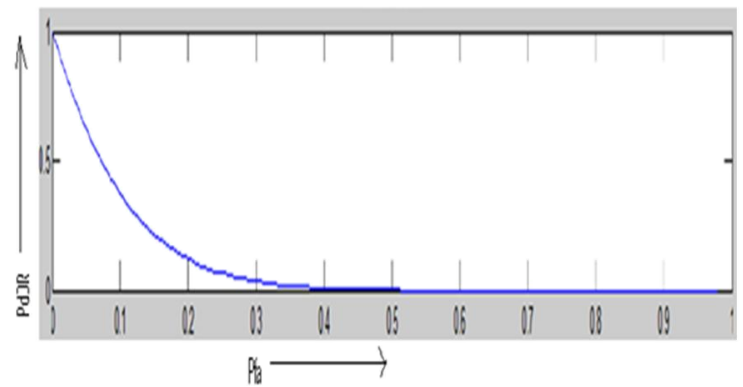

Fig, 4. Pfa vs Pd OR for Bandwidth factor 1200

Then in the following Figure. (4), we see that under OR rule, probability of miss detection in a transmission way decreases with the increase of probability of false alarm (Pfa).

It shows that this curve gives the opposite performance of AND rule. So here the detection probability is decreasing with the false alarm and it becomes zero with the increasing of false alarm.

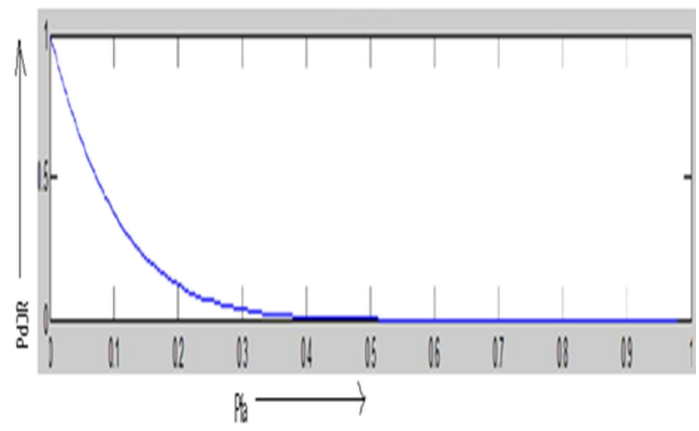

Fig. 5. Pfa vs Pd OR for Bandwidth factor 2000

In the Figure (5) we have changed the time bandwidth factor from 1200 to 2000 for the same user and SNR value by using OR rule.

Same as before we can see that for the change of time bandwidth factors there is no effect on the probability of detection with the change of probability of false alarm.

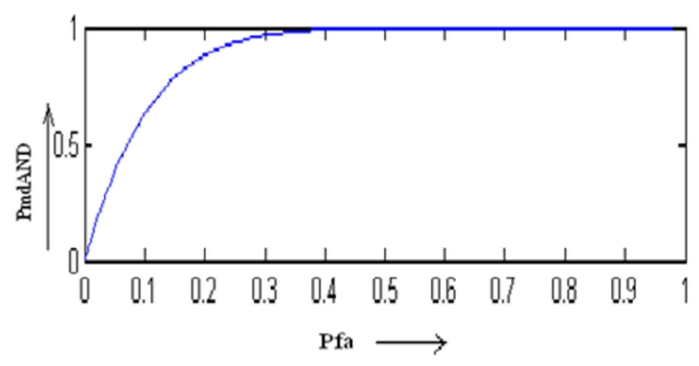

Fig. 6. Pfa vs Pmd AND for bandwidth 1200
In the Figure (6), The given SNR value is $15 \mathrm{~dB}$ and the time bandwidth factor is 1200 . Here with the increase of probability of false alarm (Pfa) under AND rule, initially probability of detection in a transmission way will increase. But after some time it becomes a steady state.

We know that when the energy consumption of the cognitive radio network increases the performance and consumption also generally saturates.

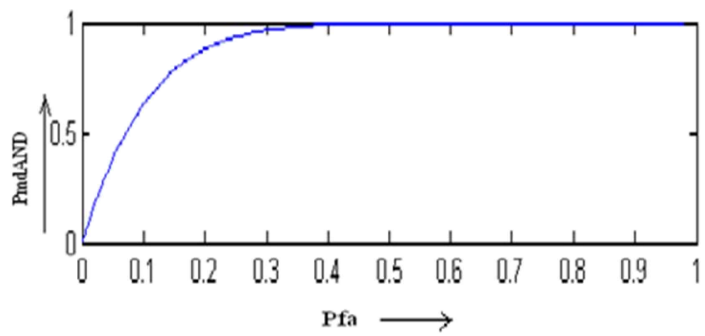

Fig. 7. Pfa vs Pmd AND for bandwidth 2000

Here in Figure (7), the probability of detection saturates within the consumption saturation. We have changed the time bandwidth factor from 1200 to 2000 for the same user and SNR value by using AND rule.

We can see that for the change of time bandwidth factors there is no effect on the probability of miss detection.

So, from these figures we see that for the fixed bandwidth factor AND rule gives a better probability detection of unused spectrum than OR, Majority rules.

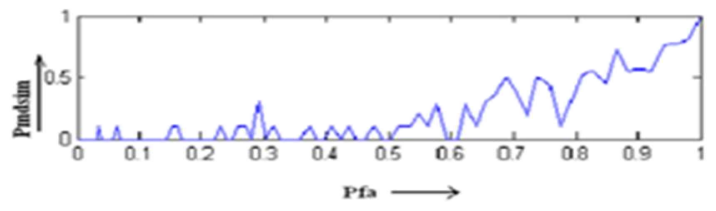

Fig. 8. Pfa vs Pmd Andsim for CR user 10

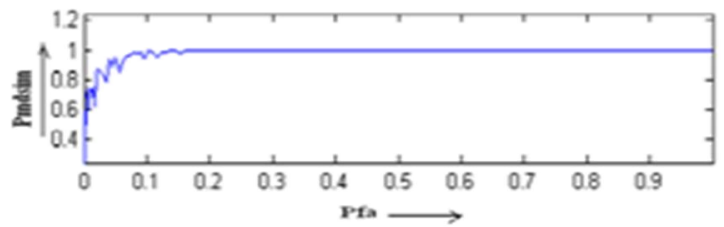

Fig. 9. Pfa vs Pmd Andsim for CR user 20

After that we want to see the effect of detection probability by varying the cognitive user numberin Figure (8). We observed the plot for the probability 
L. Nahar et al. / International Journal of Computer Networks and Communications Security, 1 (6), November 2013

of detection for minimum number of users 10 under the AND rule. The value of probability of miss detection for a given value of probability of false alarm increases in a zigzag curve with the minimum number of users. Here the detection probability could not be found out significantly.

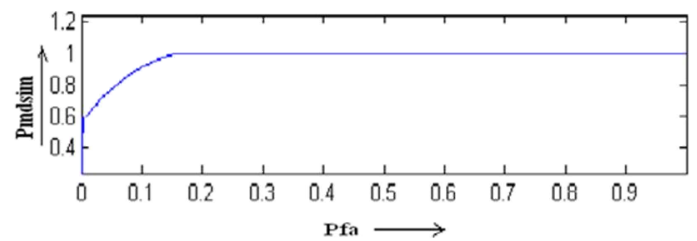

Fig. 10. Pfa vs Pmd Andsim for CR user 40

When we increased the number of cognitive users, we found that there is a change of probability of detection in the transmission way which can be seen in Figure (9) and Figure (10).

Here we observed the increasing number of users gives better performance for probability of detection than the minimum user. Here a group of cooperating CR users performs local sensing with an RF front end and a local processing unit. By increasing $\mathrm{CR}$ users the unused spectrum decreases within the RF environment. So the increasing user number of detecting unused spectrum and sharing it, without harmful interference to other users which provides better performance for probability of detection.

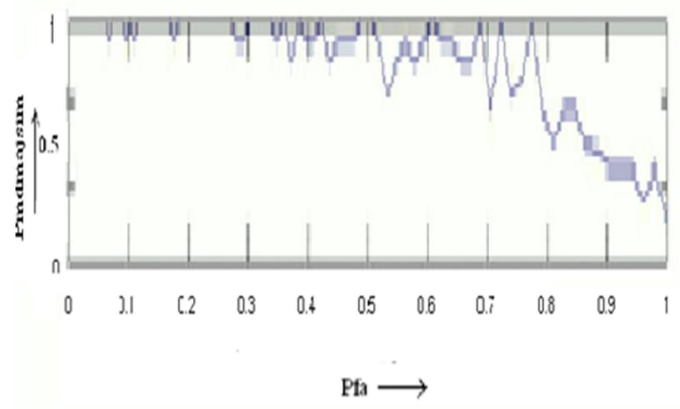

Fig. 11. Pfa vs Pdmajsim for CR user 10

In Figure (11) we simulate the miss detection of user for Majority rule. Here we see that the detection probability for sensing spectrum decreases with false alarm.

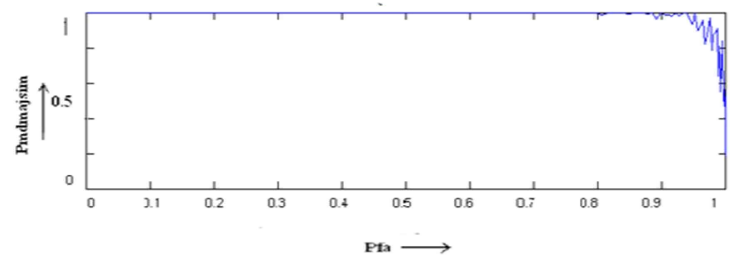

Fig. 12. Pfa vs Pdmajsim for CR user 40
In Figure (12) when we increase the user number then the detection probability decreases smoothly. Unused spectrum sensing under this rule is decreasing. So we cannot use this rule for sense the unused spectrum efficiently.

We also observed the impacts of detection probability for varying cognitive users.

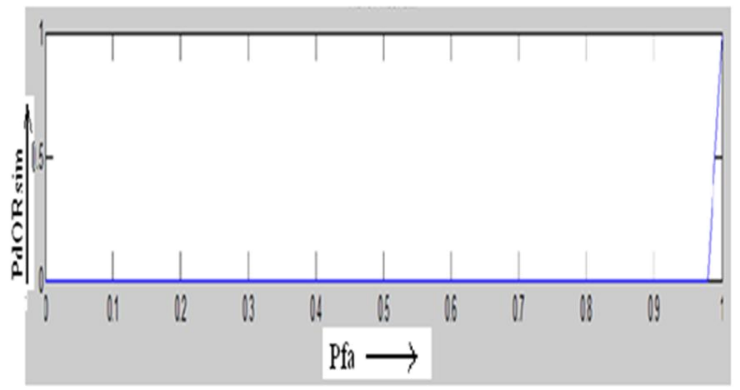

Fig. 13. Pfa vs Pd OR sim for CR user 10

First we see the effect of probability of detection for Cognitive user 10. In figure. (13) we see that when the probability of false alarm becomes very high then it gives value for the SIM detection of the user. Otherwise it becomes zero.

Then we increase the number of users and observe the impacts of detection probability for sensing spectrum.

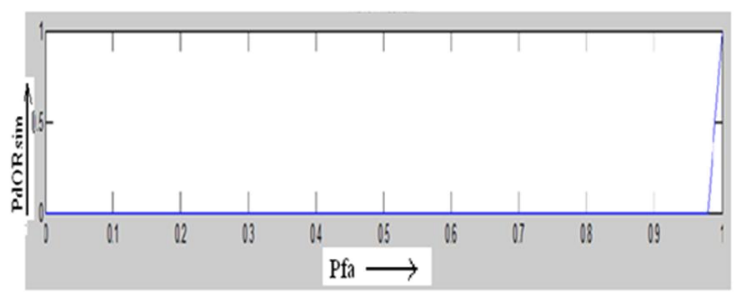

Fig. 14. Pfa v's Pd OR SIM for CR user 40

In Figure (14) we see that due to the increasing number of cognitive users the curve under OR rule remain same because in OR rule if any one of the user detect the signal then the probability of detection will be 1 . So in this rule, misuse of the user usually occurs.

So, finally we achieved a significant value for detection probability by increasing the CR users in AND rule. That is why AND rule is more effective than OR and Majority rule to find out the detection probability of the user under the AWGN channel.

We also see the effect of cognitive radio sensing spectrum under the multipath fading channel for three rules. In fading channel the original signal and noise signal convolute with each another and generate a fading signal. 
L. Nahar et al. / International Journal of Computer Networks and Communications Security, 1 (6), November 2013

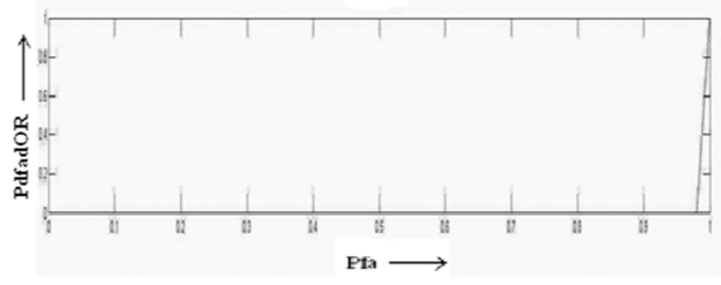

Fig. 15. Pfa vs PdfadOR for fading channel

In Figure (15) we see the fading signal detection probability for OR rule. In this figure we do not find the significant change of curvature due to low settling time effect. But there also some change in curve due to fading channel. Here in fading condition curves become desert than the curve from AWGN channel.

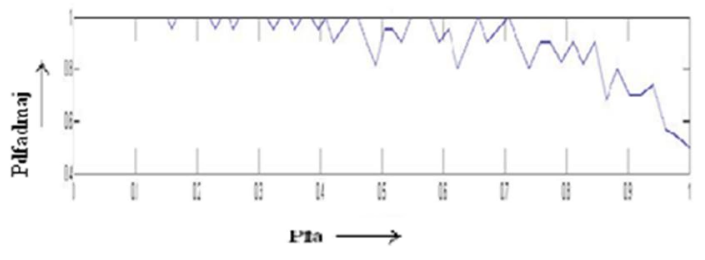

Fig. 16. Pfa vs Pdfadmajority for fading channel

After that we observe the fading signal detection probability under majority rule in fig. (16). Here the detection probability of unused spectrum also decreases. It also gives fade amplitude than the AWGN channel.

From these two figures we cannot satisfy for better sensing unused spectrum. So, finally we use the AND rule for significant value of detection probability.

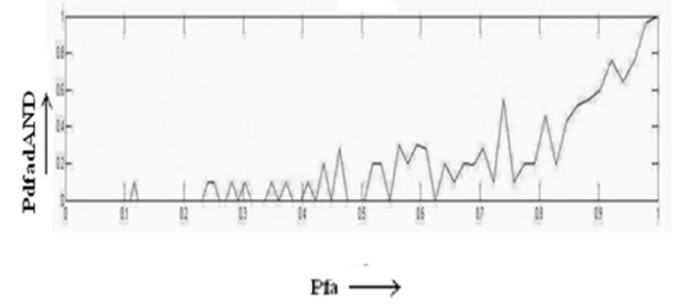

Fig. 17. Pfa vs Pmd fadAND for fading channel

From this Figure (17), we can see that the curve just fades or the amplitude becomes less than the curve of the normal AWGN channel. But the detection probability is increasing with a probability of false alarm. So we can say that in fading condition AND rule gives better detection probability than other rules. In the fading condition the signal amplitude becomes faded under three rules. In contrast among three curves we see that here also AND rule gives more significant curve. So by seeing all the curves we can say that in fading condition AND rule gives better detection probability than other rules.

\section{CONCLUSION}

We know that cooperative sensing is an effective and attractive approach to combat multiple fading and receiver's uncertainty and hidden primary problem. In cooperative technique, AND rule is employed and it evaluates the system performance by using probability of detection (Pd) and SNR as metric. Here we have shown that the AND rule gives better performance for probability detection than OR rule and Majority rule under the AWGN channel and Fading condition.

\section{REFERENCES}

[1] Sau-Hsuan $\mathrm{Wu}$, “Cognitive radio systems and experiments", IEEE Wireless Communication, vol. 6, no. issue 4, pp. 633-636, August 2008.

[2] Anugar Bansal,M.Mahajan, "Building cognitive radio system using Matlab", International Journal of Electronics and Computer Science, Vol-1, issue no. 3, pp1555-1560, November 2012.

[3] N. Armi, N.M. Saad, M. Arshad,"Hard decision fusion based cooperative spectrum sensing in cognitive radio system", ITB Journal of Information and Communication Technology, vol. 3, issue-2, pp. 109-122.2009.

\section{AUTHOR PROFILES:}

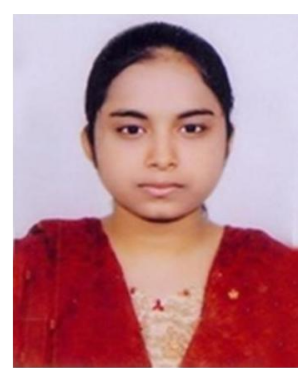

Lailun Nahar: She was born in Dhaka, Bangladesh. She has received her BSc. Degree in Electrical \& Electronic engineering with a major in Communication from East West University, Bangladesh in 2013.Her main areas of interests are networking \& communications. She is currently working as Teaching Assistant at East West University, Dhaka, Bangladesh.

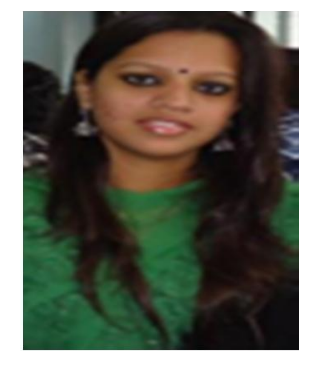

Sameha Zaman Ruthi: She was born in Naogaon, Rajshahi, Bangladesh. She has received her BSc. Degree in Electrical \& Electronic engineering with a major in Communication from East West University, Bangladesh in 2013.Her main area of 
interests is communication. She is currently doing her internship in LM Ericsson Bangladesh Ltd.

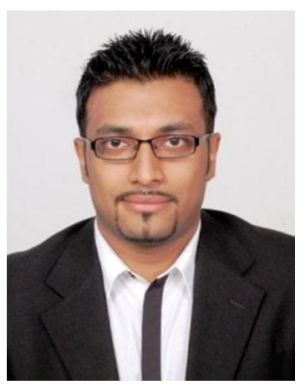

Fakir Mashuque Alamgir. He was born in Dhaka, Bangladesh. He did his B.Sc. in Computer Science \& Communication Engineering from University of Greenwich, London, UK in 2007. He received the M.Sc. degree in Wireless Communication from University of Greenwich, London, UK in 2008. His main areas of interests are networking and Communications. He is currently working as a Senior Lecturer at East West University, Dhaka, Bangladesh.

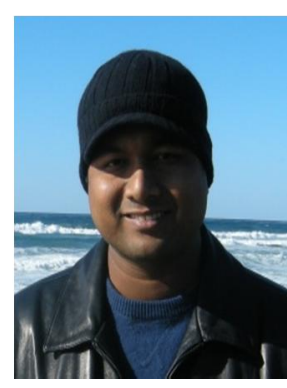

Anwarul Azim. He was born in Dhaka, Bangladesh. He did his B.Sc. in Electrical \& Electronic Engineering from Islamic University of Technology, Gazipur, Bangladesh in 2006. He received the M.Sc. degree in Electrical Engineering from Colorado State University, USA in 2010. His main areas of interests are Wireless networking and Communications. He is currently working as a Lecturer at East West University, Dhaka, Bangladesh. 